PROCEEDINGS OF THE

AMERICAN MATHEMATICAL SOCIETY

Volume 129, Number 8 , Pages 2353-2358

S 0002-9939(00)05807-X

Article electronically published on December 28, 2000

\title{
SEPARATION FOR KERNELS OF HANKEL OPERATORS
}

\author{
CAIXING GU
}

(Communicated by Joseph A. Ball)

\begin{abstract}
We prove that for two Hankel operators $H_{a_{1}}$ and $H_{a_{2}}$ on the Hardy space of the unit disk either the kernel of $H_{a_{1}}^{*} H_{a_{2}}$ equals the kernel of $H_{a_{2}}$ or the kernel of $H_{a_{2}}^{*} H_{a_{1}}$ equals the kernel of $H_{a_{1}}$. In fact we prove a version of the above result for products of an arbitrary finite number of Hankel operators. Some immediate corollaries are generalizations of the result of Brown and Halmos on zero products of two Hankel operators and the result of Axler, Chang and Sarason on finite rank products of two Hankel operators. Simple examples show our results are sharp.
\end{abstract}

\section{INTRODUCTION}

Let $L^{2}$ be the space of Lebesgue square integrable functions on the unit circle and $L^{\infty}$ be the space of essentially bounded functions on the unit circle. The Hardy space $H^{2}$ is the closed linear span of analytic polynomials in $L^{2}$. Let $P$ be the projection of $L^{2}$ onto $H^{2}$. For $f \in L^{\infty}$, the Toeplitz operator $T_{f}: H^{2} \rightarrow H^{2}$ with symbol $f$ is defined by the rule $T_{f} h=P(f h)$. The Hankel operator $H_{f}$ : $H^{2} \rightarrow L^{2} \ominus H^{2}$ with symbol $f$ is defined by $H_{f} h=(I-P)(f h)$. Let $g \in L^{\infty}$. The Toeplitz operators and Hankel operators are connected by the following important relation:

$$
T_{\bar{f} g}-T_{\bar{f}} T_{g}=H_{f}^{*} H_{g} .
$$

The Hankel operator $H_{f}$ depends only on $(I-P) f$. If $(I-P) f=\sum_{k=1}^{\infty} f_{k} \bar{z}^{k}$, then $H_{f}$ has a matrix representation (called a Hankel matrix) $\left(f_{k+l}\right)_{l \geq 1, k \geq 0}$ with respect to bases $\left\{z^{k}\right\}_{k=0}^{\infty}$ of $H^{2}$ and $\left\{\bar{z}^{l}\right\}_{l=1}^{\infty}$ of $L^{2} \ominus H^{2}$.

The present work is motivated by a problem on finite rank products of Hankel operators. The classical Kronecker's result [4] on finite Hankel rank matrices is that $H_{f}$ is of finite rank if and only if $(I-P) f$ is a rational function, and in this case the rank is equal to the degree of $(I-P) f$. Here we recall that if $r=p / q$ for polynomials $p$ and $q$, the degree of $r$ is the maximum of the degree of $p$ and the degree of $q$. Axler, Chang and Sarason [1] proved, among other things, that

$$
\operatorname{rank}\left(H_{f}^{*} H_{g}\right)=\min \{\operatorname{deg}((I-P) f), \operatorname{deg}((I-P) g)\} .
$$

Received by the editors May 4, 1999 and, in revised form, December 7, 1999.

2000 Mathematics Subject Classification. Primary 47B35.

Key words and phrases. Hankel operator.

This research was partially supported by the National Science Foundation Grant DMS-9706838 and the SFSG Grant of California Polytechnic State University. 
In other words $H_{f}^{*} H_{g}$ is of finite rank if and only if either $H_{f}$ or $H_{g}$ is of finite rank, and in this case the rank of $H_{f}^{*} H_{g}$ is equal to the minimum of the rank of $H_{f}$ and the rank of $H_{g}$. The proof of the above result is elegant and uses Beurling's invariant subspace theorem. A purely algebraic proof was given by Richman [6]. He does not assume that $H_{f}$ and $H_{g}$ are bounded, so his result is slightly stronger. As long as $H_{f}^{*} H_{g}$ is a bounded operator, the same result holds. He showed that the analogue of his result for products of three Hankel matrices does not hold by displaying three Hankel matrices (not all are bounded) whose product is zero. He raised the question of whether or not the rank of the product of three bounded Hankel operators is equal to the minimum of the ranks of the individual ones.

Xia and Zheng [7] discussed zero or compact products of three Hankel operators. Let $\theta_{i}, i=1,2,3$, be three inner functions. They proved that $H_{\overline{\theta_{1}}} H_{\overline{\theta_{2}}} H_{\overline{\theta_{3}}}$ is zero if and only if $\theta_{1} \theta_{3}$ divides $\theta_{2}-\lambda$ for some constant $\lambda$. For example, $H_{\bar{z}^{2}} H_{\bar{z}^{3}} H_{\bar{z}}=0$. This answers negatively Richman's question.

Let $a_{i} \in L^{\infty}$ for $i=1, \cdots, n$ throughout the paper. In [3] we characterize when a product of four Hankel operators is of finite rank and use this characterization to prove that

$$
\max _{\sigma}\left\{\operatorname{rank}\left(H_{a_{\sigma(1)}}^{*} H_{a_{\sigma(2)}} H_{a_{\sigma(3)}}^{*} H_{a_{\sigma(4)}}\right)\right\}=\min _{1 \leq i \leq 4} \operatorname{rank}\left(H_{a_{i}}\right)
$$

where the maximum is taken over all permutations $\sigma$ of $\{1,2,3,4\}$. A similar rank formula holds for products of three Hankel operators.

In this paper we extend the above formula to products of an abitrary finite number of Hankel operators by proving a separation principle for kernels of products of Hankel operators, which is interesting in itself. Let $S_{n}$ be the permutation group of $n$ integers $\{1, \cdots, n\}$. For notational convienence, let

$$
H\left(a_{\sigma(1)}, \cdots, a_{\sigma(n)}\right)=H_{a_{\sigma(1)}}^{*} H_{a_{\sigma(2)}} \cdots H_{a_{\sigma(2 k-1)}}^{*} H_{a_{\sigma(2 k)}}
$$

if $n=2 k$ is even and

$$
H\left(a_{\sigma(1)}, \cdots, a_{\sigma(n)}\right)=H_{a_{\sigma(1)}} H_{a_{\sigma(2)}}^{*} \cdots H_{a_{\sigma(2 k-1)}} H_{a_{\sigma(2 k)}}^{*} H_{a_{\sigma(2 k+1)}}
$$

if $n=2 k+1$ is odd.

Theorem 1.1. There exists a permutation $\sigma \in S_{n}$ such that the kernel of $H\left(a_{\sigma(1)}, \cdots, a_{\sigma(n)}\right)$ is equal to the kernel of $H_{a_{\sigma(n)}}$.

For an operator $A, A$ is of finite rank if and only if the kernel of $A$ is of finite codimension, and in this case the rank of $A$ is equal to the codimension of the kernel of $A$. Thus we have the following result.

Corollary 1.2. Using the notation as above, we have

$$
\max _{\sigma \in S_{n-1}} \operatorname{rank}\left[H\left(a_{\sigma(1)}, \cdots, a_{\sigma(n-1)}, a_{n}\right)\right]=\min _{1 \leq i \leq n} \operatorname{rank}\left(H_{a_{i}}\right) .
$$

Corollary 1.3. If $H\left(a_{\sigma(1)}, \cdots, a_{\sigma(n-1)}, a_{n}\right)=0$ for all permutations $\sigma \in S_{n-1}$, then one of $H_{a_{i}}$ is zero.

For $n=2$ in Corollary [1.3, this is the result of Brown and Halmos [2]. For $n=3$ in Corollary 1.3] this result is slightly stronger than Theorem 3.3 in the paper [7] by Xia and Zheng. 


\section{The MAIN RESUlT}

For an operator $A, \operatorname{Ker}(A), \operatorname{Range}(A)$ and $\operatorname{rank}(A)$ denote the kernel, range and rank of $A$, respectively $(\operatorname{rank}(A)=\infty$ if $A$ is not a finite rank operator). For an inner funtion $\theta \in H^{2}$, let $\mathcal{H}(\theta)=H^{2} \ominus \theta H^{2}$.

The following lemma is well known (see [5] for example).

Lemma 2.1. $\operatorname{Ker}\left(H_{f}\right) \neq\{0\}$ if and only if $f$ is of the form $\bar{\theta} b$ where $\theta$ is some inner function and $b \in H^{\infty}$ has the property that the inner part of $b$ and $\theta$ are coprime. Furthermore we have:

1. $\operatorname{Ker}\left(H_{\bar{\theta} b}\right)=\theta H^{2}, \quad \operatorname{Ker}\left(H_{\bar{\theta} b}^{*}\right)=\overline{z \theta H^{2}}$,

2. $\operatorname{closure}\left\{\operatorname{Range}\left(H_{\bar{\theta} b}^{*}\right)\right\}=\left\{\operatorname{Ker}\left(H_{\bar{\theta} b}\right)\right\}^{\perp}=H^{2} \ominus \theta H^{2}=\mathcal{H}(\theta)$,

3. $\operatorname{closure}\left\{\operatorname{Range}\left(H_{\bar{\theta} b}\right)\right\}=\overline{z \mathcal{H}(\theta)}$.

Lemma 2.2. Let $\sigma \in S_{n}$ be the permutation such that $\sigma(1)=n$ and $\sigma(i)=i-1$ for $2 \leq i \leq n$. Let $C$ be any bounded linear operator with range in $H^{2}$. There exists an $i(1 \leq i \leq n)$ such that

$$
\operatorname{Ker}\left(H_{a_{\sigma(i)}}^{*} H_{a_{i}} C\right)=\operatorname{Ker}\left(H_{a_{i}} C\right)
$$

Similarly, there exists an $i(1 \leq i \leq n)$ such that

$$
\operatorname{Ker}\left(H_{a_{\sigma(i)}} H_{a_{i}}^{*} D\right)=\operatorname{Ker}\left(H_{a_{i}}^{*} D\right)
$$

where $D$ is any bounded linear operator with range in $L^{2} \ominus H^{2}$.

Proof. It is clear that if $\operatorname{Ker}\left(H_{a_{i}}^{*}\right)=0$ for some $i$, we are done. Therefore by Lemma 2.1 we can assume that for $i=1, \cdots, n, a_{i}=\overline{\theta_{i}} b_{i}$, where $\theta_{i}$ is inner and $b_{i} \in H^{\infty}$ has the property that the inner part of $b_{i}$ and $\theta_{i}$ are coprime. Let $C$ be a bounded linear operator from a complex Hilbert space $K$ into $H^{2}$. We prove the lemma by contradiction. If (2.1) does not hold, let $p_{i}$ be non-zero such that $p_{i}=C d_{i}$ for some $d_{i} \in K$ and

$$
p_{i} \in \operatorname{Ker}\left(H_{a_{\sigma(i)}}^{*} H_{a_{i}}\right) \ominus \theta_{i} H^{2}, \quad i=1, \cdots, n .
$$

Thus $H_{a_{i}} p_{i} \in \operatorname{Ker}\left(H_{a_{\sigma(i)}}^{*}\right)$. By Lemma 2.1

$$
\overline{\theta_{i}} b_{i} p_{i}=\overline{z \theta_{\sigma(i)} h_{i}}+k_{i}, \quad i=1, \cdots, n,
$$

for some analytic functions $h_{i}, k_{i} \in H^{2}$. Rewrite the above equations as

$$
b_{i} p_{i}-\theta_{i} k_{i}=\theta_{i} \overline{\theta_{\sigma(i)} z h_{i}}
$$

for $i=2, \cdots, n$. Taking products of both sides of above $n$ equations gives

$$
\begin{aligned}
\prod_{i=1}^{n}\left(b_{i} p_{i}-\theta_{i} k_{i}\right) & =\prod_{i=1}^{n}\left(\theta_{i} \overline{\theta_{\sigma(i)} z h_{i}}\right) \\
& =\left[\prod_{i=1}^{n}\left(\theta_{i} \overline{\theta_{\sigma(i)}}\right)\right]\left[\bar{z}^{n} \prod_{i=1}^{n} \overline{h_{i}}\right]
\end{aligned}
$$

Note that $\prod_{i=1}^{n}\left(\theta_{i} \overline{\theta_{\sigma(i)}}\right)=1$. We have

$$
\prod_{i=1}^{n}\left(b_{i} p_{i}-\theta_{i} k_{i}\right)=\bar{z}^{n} \prod_{i=1}^{n} \overline{h_{i}} \text {. }
$$


Note that the left side of the above equation belongs to $z H^{2}$ and the right side of the above equation belongs to $\overline{z H^{2}}$. Therefore

$$
\prod_{i=1}^{n}\left(b_{i} p_{i}-\theta_{i} k_{i}\right)=0 .
$$

That is, $b_{i} p_{i}-\theta_{i} k_{i}=0$ for some $i \geq 1$. Since the inner part of $b_{i}$ and $\theta_{i}$ are comprime, we have $p_{i} \in \theta_{i} H^{2}$ which is a contradiction. This completes the proof.

Let

$$
J\left(a_{1}, \cdots, a_{n}\right)=H_{a_{\sigma(1)}} H_{a_{\sigma(2)}}^{*} \cdots H_{a_{\sigma(2 k-1)}} H_{a_{\sigma(2 k)}}^{*}
$$

if $n=2 k$ is even and

$$
J\left(a_{1}, \cdots, a_{n}\right)=H_{a_{\sigma(1)}}^{*} H_{a_{\sigma(2)}} \cdots H_{a_{\sigma(2 k)}} H_{a_{\sigma(2 k+1)}}^{*}
$$

if $n=2 k+1$ is odd.

The following is the proof of Theorem 1.1

Proof. We will prove a slightly stronger version of Theorem 1.1. Let $C$ be any bounded linear operator with range in $H^{2}$ and $D$ be any bounded linear operator with range in $L^{2} \ominus H^{2}$. We will show that there exists a permutation $\sigma \in S_{n}$ such that the kernel of $H\left(a_{\sigma(1)}, \cdots, a_{\sigma(n)}\right) C$ is equal to the kernel of $H_{a_{\sigma(n)}} C$, and similarly there exists a permutation $\sigma \in S_{n}$ such that the kernel of $J\left(a_{\sigma(1)}, \cdots, a_{\sigma(n)}\right) D$ is equal to the kernel of $H_{a_{\sigma(n)}}^{*} D$. We prove the result by induction on $n$. By Lemma 2.2, it is true for $n=2$. Assume it is true for $n=2 k$. For each $i=1, \cdots, 2 k$, let $\sigma_{i}$ be a permutation of $2 k$ integers $\{1,2, \cdots, i-1, i+1, \cdots, 2 k+1\}$, such that

$$
\begin{aligned}
\operatorname{Ker}\left[J \left(a_{\sigma_{i}(1)}, a_{\sigma_{i}(2)}, \cdots\right.\right. & \left.\left., a_{\sigma_{i}(i-1)}, a_{\sigma_{i}(i+1)}, \cdots, a_{\sigma_{i}(2 k)}, a_{\sigma_{i}(2 k+1)}\right) H_{a_{i}} C\right] \\
& =\operatorname{Ker}\left[H_{a_{\sigma_{i}(2 k+1)}^{*}}^{*} H_{a_{i}} C\right],
\end{aligned}
$$

and similarly let $\sigma_{2 k+1}$ be a permutation of $2 k$ integers $\{1,2, \cdots, 2 k\}$ such that

$$
\begin{gathered}
\operatorname{Ker}\left[J\left(a_{\sigma_{2 k+1}(1)}, a_{\sigma_{2 k+1}(2)}, \cdots, a_{\sigma_{2 k+1}(2 k)}\right) H_{a_{2 k+1}} C\right] \\
=\operatorname{Ker}\left[H_{a_{\sigma_{2 k+1}(2 k)}^{*}}^{*} H_{a_{2 k+1}} C\right] .
\end{gathered}
$$

Let $\delta_{i}=\sigma_{i}(2 k+1)$ for $i=1, \cdots, 2 k$ and $\delta_{2 k+1}=\sigma_{2 k+1}(2 k)$. We claim that there exists an $i, 1 \leq i \leq 2 k+1$, such that

$$
\operatorname{Ker}\left[H_{a_{\delta_{i}}}^{*} H_{a_{i}} C\right]=\operatorname{Ker}\left[H_{a_{i}} C\right] .
$$

We first show that there exists a subset $S=\left\{\alpha_{i}, i=1,2, \cdots, m\right\}$ of $\{1, \cdots, 2 k+1\}$ such that the set of all pairs of integers of the form $\left\{\left(\delta_{i}, i\right), i=1, \cdots, 2 k+1\right\}$ contains a subset of pairs of integers of the form $\left\{\left(\alpha_{i}, \beta_{i}\right): \alpha_{i}, \beta_{i} \in S, i=1, \cdots, m\right\}$ and $\alpha_{i} \neq \alpha_{j}, \beta_{i} \neq \beta_{j}$ for $i \neq j$.

Let $S_{1}$ be the set of all distinct members of $\left\{\delta_{i}, i=1, \cdots, 2 k+1\right\}$. Since $\delta_{i} \neq i, S_{1}$ contains at least two integers. Without loss of generality, let $S_{1}=$ $\left\{\delta_{i}, i=1, \cdots, m_{1}\right\}$. It is easy to see that the set of all pairs of integers of the form $\left\{\left(\delta_{i}, i\right), i=1, \cdots, 2 k+1\right\}$ contains a subset of pairs of integers of the form $\left\{\left(\alpha_{i}, \delta_{i}\right): \alpha_{i} \in S_{1}, i=1, \cdots, m_{1}\right\}$. If $\alpha_{i} \neq \alpha_{j}$ for all $i \neq j$, we are done. Otherwise let $S_{2}$ be the set of all distinct members of $\left\{\alpha_{i}, i=1, \cdots, m_{1}\right\}$ and repeat the argument as above. It is easy to see that continuing this process will yield the desired set $S$. 
Let $\beta$ denote the permutation of $m$ integers in $S$ which maps $\alpha_{i}$ to $\beta_{i}$ for $i=$ $1, \cdots, m$. Without loss of generality, assume $\beta$ is a cycle (othewise take any cycle of $\beta)$. That is, $\beta\left(\alpha_{i}\right)=\beta_{i}=\alpha_{i+1}$ for $i=1, \cdots, m-1$ and $\beta\left(\alpha_{m}\right)=\beta_{m}=\alpha_{1}$. Now again by Lemma [2.2, there exists an $i$ such that

$$
\operatorname{Ker}\left[H_{a_{\alpha_{i}}}^{*} H_{a_{\beta\left(\alpha_{i}\right)}} C\right]=\operatorname{Ker}\left[H_{a_{\beta\left(\alpha_{i}\right)}} C\right] .
$$

This proves that there exists a permutation $\sigma \in S_{2 k+1}$ such that the kernel of $H\left(a_{\sigma(1)}, \cdots, a_{\sigma(2 k+1)}\right) C$ is equal to the kernel of $H_{a_{\sigma(2 k+1)}} C$. Similarly we can show that there exists a permutation $\sigma \in S_{2 k+1}$ such that the kernel of

$$
J\left(a_{\sigma(1)}, \cdots, a_{\sigma(2 k+1)}\right) D
$$

is equal to the kernel of $H_{a_{\sigma(2 k+1)}}^{*} D$. This completes the proof.

Next we give the proof of Corollary 1.2.

Proof. First we prove the corollary for the case $n=2$. By Theorem 1.1 with $n=2$, either the kernel of $H_{a_{1}}^{*} H_{a_{2}}$ is equal to the kernel of $H_{a_{2}}$ or the kernel of $H_{a_{2}}^{*} H_{a_{1}}$ is equal to the kernel of $H_{a_{1}}$. By the fact that an operator is of finite rank if and only if its kernel is of finite codimension, and in this case the rank is equal to the codimension of its kernel, we have that either the rank of $H_{a_{1}}^{*} H_{a_{2}}$ is equal to the rank of $H_{a_{2}}$, or the rank of $H_{a_{2}}^{*} H_{a_{1}}$ is equal to the rank of $H_{a_{1}}$. But $H_{a_{1}}^{*} H_{a_{2}}$ is the adjoint of $H_{a_{2}}^{*} H_{a_{1}}$. Thus the rank of $H_{a_{1}}^{*} H_{a_{2}}$ is equal to the minimum of the rank of $H_{a_{1}}$ and the rank of $H_{a_{2}}$.

For the general case, by the proof of Theorem 1.1, there exists a permutation $\sigma \in S_{n-1}$ such that the rank of $H\left(a_{\sigma(1)}, \cdots, a_{\sigma(n-1)}, a_{n}\right)$ is equal to the rank of $H_{a_{\sigma(n-1)}}^{*} H_{a_{n}}$. By the previous analysis, the rank of $H_{a_{\sigma(n-1)}}^{*} H_{a_{n}}$ is equal to the minimum of the rank of $H_{a_{\sigma(n-1)}}$ and the rank of $H_{a_{n}}$. This proves that the left side of the rank formula in Corollary 1.2 is larger than or equal to the right side. It is obvious that the left side is less than or equal to the right side. This completes the proof.

\section{EXAMPLES}

The following simple examples show that our results are the best possible.

Example 3.1. Let $\left\{k_{i}\right\}_{i \geq 1}$ be the Fibonacci sequence, i.e., $k_{1}=1, k_{2}=2$ and $k_{i}=k_{i-1}+k_{i-2}$ for $i \geq 3$. Let $a_{i}=\bar{z}^{k_{i}}$ for $i=1, \cdots, n$. Note that $H_{\bar{z}^{i}} H_{\bar{z}^{k}}^{*} H_{\bar{z}^{j}}=0$ for all $i, j$ and $k$ such that $i+j \leq k$. Therefore $H\left(a_{\sigma(n)}, a_{\sigma(n-1)}, \cdots, a_{\sigma(2)}, a_{1}\right)$ is zero for all permutations $\sigma$ of $\{n, n-1, \cdots, 2\}$ except for the identity permutation (i.e., $\sigma(i)=i$ for $i=n, n-1, \cdots, 2$ ). This shows that the results in Corollary 1.2 and Corollary 1.3 are sharp. That is, if we replace the set $S_{n-1}$ by any proper subset of $S_{n-1}$, both Corollary 1.2 and Corollary 1.3 are false.

Example 3.2. Notation is the same as in Example 3.1. It is clear that the kernel of $H\left(a_{n}, a_{n-1}, \cdots, a_{1}\right)$ is equal to the kernel of $H_{a_{1}}$. We claim that the kernel of $H\left(a_{\sigma(n)}, a_{\sigma(n-1)}, \cdots, a_{\sigma(1)}\right)$ is not equal to the kernel of $H_{a_{\sigma(1)}}$ for all non-trivial permutations of $\{n, n-1 \cdots, 2,1\}$. This shows that the result in Theorem 1.1 is also sharp. To see this, note that $H\left(a_{\sigma(n)}, a_{\sigma(n-1)}, \cdots, a_{\sigma(1)}\right)$ is zero unless $\sigma(n)=n$ or $\sigma(1)=n$.

If $\sigma(1)=n$, it is easy to see that the kernel of $H\left(a_{\sigma(n)}, a_{\sigma(n-1)} \cdots, a_{\sigma(1)}\right)$ is not equal to the kernel of $H_{a_{n}}$, since

$$
H\left(a_{\sigma(n)}, a_{\sigma(n-1)}, \cdots, a_{\sigma(2)}, a_{n}\right) 1=0, \quad H_{a_{n}} 1=\bar{z}^{k_{n}} \neq 0 .
$$


Therefore $\sigma(n)=n$. Similar arguments show that in order for the kernel of

$H\left(a_{\sigma(n)}, a_{\sigma(n-1)}, \cdots, a_{\sigma(1)}\right)$ to equal the kernel of $H_{a_{\sigma(1)}}$, we must have $\sigma(i)=i$ for $i=n, n-1, \cdots, 2,1$.

\section{REFERENCES}

[1] S. Axler, S.-Y. A. Chang, D. Sarason, Product of Toeplitz operators, Integral Equations and Operator Theory 1 (1978), 285-309. MR 80d:47039

[2] A. Brown and P. R. Halmos Algebraic properties of Toeplitz operators, J. Reine Angew. Math. 213 (1963), 89-102. MR 28:3350

[3] C. Gu, Finite rank products of four Hankel operators, Houston Journal of Mathematics 25 (1999), 543-561. CMP 2000:06

[4] L. Kronecker, Zur Theorie der Elimination einer Variablen aus zwei algebraischen Gleichungen, Montasber. Königl. Preussischen Acad Wies, Berlin, 1881, pp. 535-600.

[5] N.K. Nikolski, Treatise on the Shift operators, Springer-Verlag, 1986. MR 87i:47042

[6] D.R. Richman, A new proof of a result about Hankel operators, Integral Equations and Operator Theory $\mathbf{5}$ (1982), 892-900. MR 84d:47035

[7] D. Xia and D. Zheng, Products of Hankel operators, Integral Equations and Operator Theory 29 (1997), 339-363. MR 99i:47049

Department of Mathematics, California Polytechnic State University, San Luis Obispo, California 93407

E-mail address: cgu@calpoly.edu 